To appear in the Astrophysical Journal.

Preprint typeset using $\mathrm{AT}_{\mathrm{E} X} \mathrm{X}$ style emulateapj v. 01/23/15

\title{
MERIDIONAL CIRCULATION IN THE SOLAR CONVECTION ZONE: TIME-DISTANCE HELIOSEISMIC INFERENCES FROM FOUR YEARS OF HMI/SDO OBSERVATIONS
}

\author{
S.P. RAJAGURU \\ Indian Institute of Astrophysics, Koramangala II Block, Bangalore 560034, India. \\ AND \\ H.M. ANTIA \\ Tata Institute of Fundamental Research, Homi Bhabha Road, Mumbai 400005, India \\ To appear in the Astrophysical Journal.
}

\begin{abstract}
We present and discuss results from time-distance helioseismic measurements of meridional circulation in the solar convection zone using 4 years of Doppler velocity observations by the Helioseismic and Magnetic Imager (HMI) onboard the Solar Dynamics Observatory (SDO). Using an in-built mass conservation constraint in terms of the stream function we invert helioseismic travel times to infer meridional circulation in the solar convection zone. We find that the return flow that closes the meridional circulation is possibly beneath the depth of $0.77 R_{\odot}$. We discuss the significance of this result in relation to other helioseismic inferences published recently and possible reasons for the differences in the results. Our results show clearly the pitfalls involved in the measurements of material flows in the deep solar interior given the current limits on signal-to-noise and our limited understanding of systematics in the data. We also discuss the implications of our results for the dynamics of solar interior and popular solar dynamo models.
\end{abstract}

Keywords: Sun: helioseismology — Sun: interior — Sun: oscillations

\section{INTRODUCTION}

The structure and dynamics of large-scale material flows on the solar surface and in the convection zone play fundamental roles in the working of solar dynamo and its cyclic variation (Charbonneau 2010). Circulating currents in planes through the meridians inside a rotating star were originally proposed by Eddington (1925) as a consequence of radiative equilibrium (von Zeipel 1924) and as those responsible for the development of differential rotation. A rigorous theoretical treatment of the origin and maintenance of meridional circulation (MC) requires dynamical models that include energy and momentum transfer between convection, differential rotation and thermal stratification (Gilman \& Miller 1986; Miesch \& Toomre 2009). Mean-field hydrodynamical models of global axisymmetric flows and heat transport have been studied (Kitchatinov \& Rüdiger 1999; Kitchatinov \& Olemskov 2011); these models calculate interior differential rotation and MC. However, models with sufficient realism so as to reliably predict the deep structure of MC, with constraints from the helioseismically well determined solar interior rotation, are not yet established, although, detailed studies are in the offing (see Featherstone \& Miesch (2015) and references therein).

Observational studies, however, have led to considerable details on the velocity amplitudes (typically of $10-$ $20 \mathrm{~m} \mathrm{~s}^{-1}$ ) and variation of surface and near-surface part of MC (Komm et al. 1993; Hathawav 1996; Haber et al. 2002; Ulrich 2010; Hathaway \& Rightmire 2010), and on its contribution to the transport of magnetic flux from lower latitudes to the poles (Wang et al. 1989). This latter aspect of MC, with a modeled mass-conserving amplitude for a return flow typically near the base of the convection zone, has been a key dynamical component in the flux transport dynamo models (Choudhuri et al. 1995; Dikpati \& Charbonneau 1999). Precisely for the above reasons, reliable observational (helioseismic) inferences of the deep structure of MC is of paramount importance for understanding the origin and drivers of solar variability on cyclic time-scales.

Meridional flows on the surface have been measured using several different techniques: directly from the Doppler observations of the surface motions (Hathawav 1996; Ulrich 2010), correlation tracking of surface features such as small-scale magnetic elements (Komm et al. 1993; Hathaway \& Rightmire 2010), and local helioseismic measurements (Giles et al. 1997; Schou \& Bogart 1998; Basu et al. 1999; Haber et al. 2000, 2002; González et al. 2008; Basu \& Antia 2010). Almost all these different measurements give typical amplitudes of $10-20 \mathrm{~m} \mathrm{~s}^{-1}$ for the surface poleward flow with peak values over the latitude range of $30-50^{\circ}$. Of the above only the helioseismic analyses have provided maps of flows in the sub-surface layers. While the ring diagram analyses cover sub-surface layers to a maximum depth of about $20 \mathrm{Mm}$, the time-distance helioseismic technique (Duvall et al. 1993) can cover the flow in deeper layers. A first attempt application of timedistance helioseismology (Giles et al. 1997) to measure MC using the SOHO/MDI Doppler observations indicated that the poleward flows extended from the surface down to about $27 \mathrm{Mm}$ with a roughly constant magnitude. Since then, while the ring diagram analyses have studied in detail the MC in the near-surface layers including its temporal (solar-cycle scale) variations (González et al. 2008; Basu \& Antia 2010), a recent major development in the application of time-distance helioseismology has been the identification and removal of a large systematic center-to-limb effect (cf. Section 2) in 
the measured travel times of acoustic waves (Zhao et al. 2012, 2013). This has led to possibility of studying MC using time-distance helioseismology throughout the convection zone, especially improving the identification of flow signals in the deeper layers (Zhao et al. 2013; Jackiewicz et al. 2015). A new feature in these recent results, obtained from SDO/HMI data, has been the inference of a relatively shallow return flow starting at about $0.9 \mathrm{R}_{\odot}$ and a possible second cell of $\mathrm{MC}$ below this depth (Zhao et al. 2013). While the later study by Jackiewicz et al. (2015), using data from the Global Oscillation Network Group (GONG) observations, agrees on the shallow return flow, it has not reproduced the deeper second cell of MC. Both the above studies have used about two years of data from SDO/HMI or GONG Doppler observations. Given the high stakes that these results have on the dynamics of solar interior and the working of a large class of solar dynamo models, it is important to check the consistency and reproducibility of these results. Both of the above studies have used massconservation constraints on the inverted flows for checking the physical validity of results. Here, we improve on these results as follows: (1) we use 4 years of SDO/HMI to improve on the signal-to-noise of travel time measurements, and (2) implement an in-built mass conservation constraint in terms of stream functions in the inversion process itself, thereby consistently deriving both the horizontal (latitudinal) and radial flows.

Although, to first order, global mode frequencies are not sensitive to meridional flow, application of quasi degenerate perturbation theory (Lavely \& Ritzwoller 1992) shows that modes with close frequencies for neighbouring values of degree, $l$ can be coupled and their frequencies can be affected by meridional flows. Schad et al. (2013) have used the resulting perturbation on the eigenfunctions to study the MC in solar convection zone. Using MDI data for 2004-2010 they find evidence that MC penetrates to the base of the convection zone and the flow profile shows multiple cells in latitude and depth. This pattern is quite different from those inferred using time-distance technique and such a pattern is not seen in near surface flows which are reliably determined using the time distance and ring diagram techniques. The reason for this discrepancy is not clear, but it could be due to sensitivity of global mode perturbation to MC with multiple cells in latitude, as for multiple cells the probability of finding mode pairs (which are coupled by $\mathrm{MC}$ ) with close frequencies increases.

Another technique to estimate $\mathrm{MC}$ is to use well determined differential rotation profile in the solar convection zone and solve a hydrodynamic model including the Coriolis force and Reynolds stresses (Dikpati 2014). For a solar like density profile and differential rotation, the resulting meridional flow was found to be two-celled in latitude. Such a profile is not seen at the surface, which implies that other effects need to be included in such an analysis.

We describe the data and travel-time measurement procedure in Section 2, inversion technique in Section 3, present the results and validation tests on MC in Section 4 and discussions and conclusions in Section 5 .

\section{DATA AND ANALYSIS PROCEDURE}

\subsection{Data}

We have used the full-disk Doppler observations made by the SDO/HMI over a four year period from 2010 May 1 through 2014 April 30. The data consists of $45 \mathrm{~s}$ cadence Doppler velocities at the solar surface. The first 2 years of data are the same as those used by Zhao et al. (2013), and we have added the next 2 years' data to it. The data are tracked to account for the solar rotation and remapped using Postel's projection to have a uniform latitude and longitude grid. This process is the same as that performed by Zhao et al. (2013) using the JSOC helioseismology pipeline at Stanford. The original extracts from the JSOC database for this study used a spatial sampling scale of $0.18 \mathrm{deg} /$ pixel, and we further resample (or smooth) the data to a scale of $0.36 \mathrm{deg} / \mathrm{pixel}$ in order to reduce the volume of data handled and the computing time. This increase in pixel size (or reduction in resolution) does not make any difference to the travel time measurements over distances greater than 2 deg., and further our main focus is on deeper layers which are probed by waves skipping at the surface over distances much larger than a degree.

\subsection{Analysis Procedure}

We use the technique of time-distance helioseismology (Duvall et al. 1993) in the so called deep-focus geometry, in much the same way as that described by Zhao et al. (2013) except for implementing an improvement as discussed below. This method involves cross-correlating Doppler signals from arcs, typically 30 degree wide, cut out from an annulus of diameter equaling the surface travel distance of the helioseismic waves, whose travel times that we want to measure. These arcs are placed perpendicular to the direction over which we want to measure the flows (e.g., for meridional flows the arcs are placed perpendicular to the meridians). Ray-paths connecting diametrically opposite points in the arcs meet at the lower turning point of waves traveling a surface distance of $\Delta$ that equals the diameter, and it is also the deep-focus location. This location is directly beneath the centre point of the arcs (or annulus) at the surface, hence the measurement is assigned to this surface point. Measurements are repeated by moving the centre point to every pixel of the area that we want to cover on the solar surface. For each measurement, Zhao et al. (2013) took the average of Doppler signals over all pixels in one arc and cross-correlated it with that from the opposite arc. This averaging procedure, however, does not preserve the travel distance $(\Delta=$ the diameter) as it involves contributions from all pairs of points (or pixels) between the arcs not just the diametrically opposite ones. In other words, a travel-time measurement by Zhao et al. (2013) for a given $\Delta$ involves contributions from waves of smaller distances down to $\approx \cos \left(15^{\circ}\right) \Delta \square$, and hence measurements are biased towards lower depths at each depth, and this we believe will lead to poorer depth resolution as $\Delta$ increases, i.e. for deeper measurements.

1 This estimate holds when neglecting the spherical curvature on the solar surface, i.e. when the distances $\Delta$ are not large. Otherwise, exact expression for the smallest distance, $\alpha$, between pairs of points from opposite arcs cut from a circle of diameter $\Delta$ is given by $\alpha=2 \sin ^{-1}[\sin (\Delta / 2) \cos (\theta / 2)]$, where $\theta$ is the arc-length in degrees 
We correct for this in our measurements, but at the cost of much increased computing time, by splitting the arc into small segments (typically $1^{\circ}$ to $3^{\circ}$ segments, depending on $\Delta$ ) and cross-correlating the diametrically opposite segments [such a deep-focus geometry was originally proposed by Duvall (1995); see also Rajaguru (2008); Hanasoge et al. (2010)], and then averaging the resulting cross-correlations for a given measurement. We denote travel times estimated from such 'point-to-point' cross-correlations with a string $p-p$. We also calculate cross-correlations between arc-averaged signals, exactly replicating the procedure of Zhao et al. (2013), and the travel times from such 'arc-to-arc' cross-correlations are denoted as $a-a$ (refer to Figure 2).

The rest of our measurement procedure is identical to Zhao et al. (2013) in making one travel time measurement for each month (of four years of data that we use here): (1) a cross-correlation computation at a given location involves full-disk data cubes tracked for one day, (2) measurement is restricted to $30^{\circ}$ (in heliographic coordinates) wide strips centered about the central-meridian (for flows in the North - South direction) or the equator (for flows in the West - East direction) [the W-E travel time differences, after accounting for rotation signals, capture the centre-to-limb systematics in travel times that are to be subtracted from the N-S travel times (Zhao et al. 2012, 2013), see below], (3) each day's cross-correlation functions are averaged over the same latitudes (longitudes) for N-S (W-E), and then averaged again over one month intervals, and (4) each monthly averaged cross-correlation function is fitted by a Gabor wavelet (Kosovichev \& Duvall 1997) to estimate the acoustic wave travel times at each latitude (for N-S) or longitude (for $\mathrm{W}-\mathrm{E}$ ) in two opposite directions between the arcs. The difference between the travel times in the two opposite directions is related to the flow velocities in the region traversed by the waves (Duvall et al. 1993; Giles et al. 1997). For example, for the N-S aligned arcs, the difference $\delta \tau_{\mathrm{NS}}=\tau_{\mathrm{NS}}-\tau_{\mathrm{SN}}$ between the $\mathrm{N}-\mathrm{S}$ and $\mathrm{S}-\mathrm{N}$ travel times will be positive for a flow towards the north pole. We have employed 60 travel distances, $\Delta$, ranging between $2.16^{\circ}$ and $44.64^{\circ}$ in steps of $0.72^{\circ}$, covering a depth range from near the surface down to about $0.7 R_{\odot}$, again the same as that of Zhao et al. (2013).

As shown by Zhao et al. (2012), there is a large systematic increase in travel time differences against angular distance from the solar disk center mimicking a radial outflow from the centre towards the limb, and which increases as $\Delta$ increases. This large center-to-limb systematics (CLS) in travel times is still of unknown origin, although, the analyses of Zhao et al. (2012, 2013) involving comparisons of travel times from different observables (corresponding to different heights of formation in the solar atmosphere) from the SDO/HMI as well as with that from another instrument (SOHO/MDI) pointed to possible physical causes in the solar atmosphere related to observation height differences. A study by Baldner \& Schou (2012) showed that the near-surface granular convection could affect the wave-propagation in the observable layers leading to a similar effect as the CLS in travel times. Although, a consistent explanation of the origin of CLS in helioseismic measurements from different observables is yet to be achieved, it is clear that the CLS in travel times are not related to sub-surface flows. The empirical prescription to remove them in the travel times, as demonstrated by Zhao et al. (2012), has led to improved measurements of meridional flows.

\section{TRAVEL-TIME INVERSION TECHNIQUE}

Within the ray approximation, the travel-time difference $\delta \tau$ between the two opposite directions along a ray path $\Gamma_{0}$ is given by (Kosovichev 1996):

$$
\delta \tau=-2 \int_{\Gamma_{0}} \frac{\mathbf{u} \cdot \hat{\mathbf{n}}}{c^{2}} d s,
$$

where $\mathbf{u}$ is the flow velocity and $\hat{\mathbf{n}}$ is the unit vector along the ray path. For ray paths confined to N-S plane, only the meridional flow will contribute to the traveltime. In general the contribution from the $u_{\theta}$ component would be much larger than that from the $u_{r}$ component of the meridional flow. This is because in general, the $u_{\theta}$ component is larger than $u_{r}$ and further contribution from $u_{r}$ tends to cancel out between the rising and falling branch of the ray path, while $u_{\theta}$ contribution in the two branches add to each other.

Because of small contribution from $u_{r}$ to $\delta \tau$, it is not possible to directly determine this component from the measured travel-times. However, the two components of the meridional flow are not independent, as they satisfy the continuity equation. Thus it is possible to determine both components and to satisfy the continuity equation, if instead we determine the stream function, $\psi$, which gives:

$$
\begin{aligned}
& \rho u_{r}=\frac{1}{r} \frac{\partial \psi}{\partial \theta}+\frac{\cos \theta}{r \sin \theta} \psi, \\
& \rho u_{\theta}=-\frac{\partial \psi}{\partial r}-\frac{\psi}{r},
\end{aligned}
$$

where $\rho$ is the density in a solar model that is used to calculate the ray paths. Thus we use Eq. 1 to determine $\psi$ using the observed travel-times. Because of large variation in density, $\psi$ also shows similar variation, as a result we actually use $\psi^{\prime}=\psi / \rho$ for inversions.

We use the Regularized Least Squares (RLS) technique to solve the inverse problem to calculate $\psi^{\prime}$ in the convection zone. For this we expand $\psi^{\prime}(r, \theta)$ in terms of cubic B-splines (e.g., Antia (2012)) in $r$ and $\theta$ :

$$
\psi^{\prime}(r, \theta)=\sum_{i} \sum_{j} a_{i j} \Phi_{i}^{r}(r) \Phi_{j}^{\theta}(\theta),
$$

where $\Phi_{i}^{r}(r)$ are the cubic B-spline basis covering $0.69 R_{\odot} \leq r \leq R_{\odot}$ and $\Phi_{j}^{\theta}(\theta)$ are the cubic B-spline basis covering $|\theta-\pi / 2| \leq 1.055$. We use 38 knots in $r$ which are uniformly spaced in acoustic depth and 31 knots in $\theta$ which are uniformly spaced in $\theta$ to define the B-spline basis functions. The coefficients of expansion $a_{i j}$ are determined using RLS with second derivative smoothing (Gough \& Thompson 1991) in both $r$ and $\theta$ by minimizing the function

$$
\sum_{i}\left(\frac{d_{i}}{\sigma_{i}}\right)^{2}+\lambda_{r}^{2} \sum\left(\frac{\partial^{2} \psi^{\prime}}{\partial r^{2}}\right)^{2}+\lambda_{\theta}^{2} \sum\left(\frac{\partial^{2} \psi^{\prime}}{\partial \theta^{2}}\right)^{2},
$$

where $d_{i}$ are the residual in the fit to Eq. 10(i.e., the difference between the left hand side and right hand sides of the equation) and $\sigma_{i}$ are the corresponding errors in travel time differences. Here the summation in the last 
two terms are over all knots in the B-spline representation and $\lambda_{r}$ and $\lambda_{\theta}$ are the two smoothing parameters. Apart from smoothing we also apply the boundary condition $\psi^{\prime}=0$ at the upper boundary. In principle, the same condition should be applied at the lower boundary, if the flow is contained within the volume considered. Since we do not know the lower boundary where the flow ends, we have not applied this boundary condition. We have verified that adding this boundary condition forces $u_{r}$ to vanish at the lower boundary $\left(r=0.69 R_{\odot}\right)$ and the resulting solution is modified in the neighbourhood of the boundary, but the solution over the bulk of the region is unaffected. These smoothing parameters are chosen as the minimum values that are needed to obtain a solution that is smooth. Once these coefficients are known, $u_{r}$ and $u_{\theta}$ are easily computed over the entire interval. Because of very small density scale height in the near surface region, we encountered some difficulty in calculating $\psi^{\prime}$ in this region. This problem is probably because our knot spacing in this region is much larger than the density scale height. To avoid this we use the value of $\psi^{\prime}$ at $r=0.995 R_{\odot}$ for larger values of $r$ also. This may be justified as earlier studies do not show much variation in the meridional flow velocity above this layer. Further, we find that removing this artifact only affects the solution for $r>0.99 R_{\odot}$, which is not the region where we are primarily interested in this work.

To calculate the errors in inverted flow velocities, we repeat the calculations 100 times with $\delta \tau$ randomly perturbed with estimated errors in observed values. In addition to estimate the systematic error due to choice of smoothing parameters we also perturb these randomly. The standard deviation in these values would give an estimate of errors in inversion.

\section{RESULTS}

\subsection{Travel-time Differences due to Meridional Flows}

Travel-times of waves skipping at distances, $\Delta$, on the surface ranging between $2.16^{\circ}$ and $44.64^{\circ}$ correspond to lower turning points, $r_{t}$, between $0.987 \mathrm{R}_{\odot}$ and $0.701 \mathrm{R}_{\odot}$, thus covering the whole depth range of the convection zone. Hence, we expect that the travel time differences $\delta \tau_{\mathrm{NS}}$ that result after subtracting the CLS (estimated from $\delta \tau_{\text {WE }}$ after correcting for solar rotation) for the chosen range of $\Delta$ should have signatures of the MC in the whole of convection zone. We show $\delta \tau_{\mathrm{NS}}$ for a few selected $\Delta$ in Figure 1 . These travel times are estimated from the $p-p$ measurement scheme described in Section 2.2. As described earlier, each measurement shown in Figure 1 is an average of individual measurements that span the given small range of $\Delta$ (indicated in the panels) with a spacing $0.72 \mathrm{deg}$. and a time range of 47 months (in the four year period 2010 May 1 through 2014 April 30, we have the month of April 2012 missing in our data sets, and hence we have a total of 47 months) with one measurement for each month. The error bars represent standard errors estimated from these individual measurements. The errors in $\delta \tau_{\mathrm{NS}}$ and $\delta \tau_{\mathrm{WE}}$ are of similar magnitude. We have also removed a small offset in $\delta \tau_{\mathrm{NS}}$ resulting from the $P$ angle variation, i.e. the variation of the angle the solar rotation axis makes with respect to the instrument (spacecraft), following Giles et al. (1997) and Zhao et al. (2013).

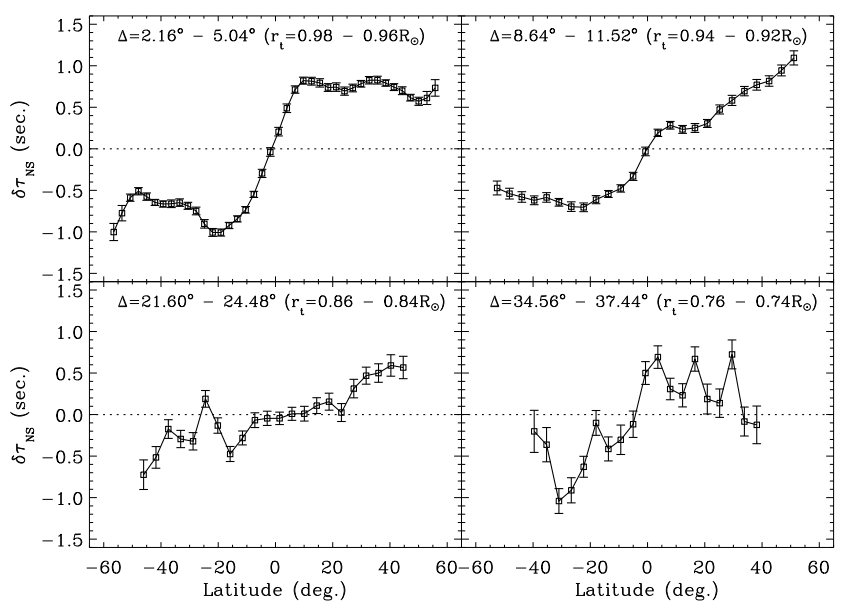

Figure 1. Travel-time differences, $\delta \tau_{\mathrm{NS}}$, against latitude for four selected travel distances $(\Delta)$. Each measurement shown here is an average of individual measurements, with a spacing $0.72 \mathrm{deg}$. in $\Delta$ and one for each of 47 months. The error bars represent standard errors estimated from these individual measurements. The centerto-limb systematics in travel times estimated through $\delta \tau_{\mathrm{WE}}$ have been subtracted from $\delta \tau_{\mathrm{NS}}$.

Variation of $\delta \tau_{\mathrm{NS}}$, averaged over three different latitude ranges, against $\Delta$ (and hence against lower turning points of p modes) is shown in Figure 2. We have shown measurements from both our improved $p-p$ measurement scheme and the $a-a$ scheme of Zhao et al. (2013). In general, we find that travel times from both these schemes agree well for the few smaller $\Delta$, but there are significant differences for $\Delta>30^{\circ}$ over low and midlatitudes. For further analyses and inversions we use only the $p-p$ travel times. We believe that the variation seen against the three latitude ranges for $\Delta>22^{\circ}$, and the North - South asymmetry in them capture the signatures of deep meridional circulation. It is interesting to note the differences in travel times against the latitude ranges used in the three panels of Figure 2, especially for large $\Delta$ : travel time averages over $30^{\circ}-40^{\circ}$ latitudes exhibit a roughly constant value of about $0.5 \mathrm{~s}$ over $15^{\circ}<\Delta<32^{\circ}\left(0.9 R_{\odot}>r_{t}>0.8 R_{\odot}\right)$ and relatively large variations for $\Delta>32^{\circ}$ (i.e. for $r_{t}<0.8 R_{\odot}$ ), especially a sharp decline (at $\Delta \approx 30^{\circ}$ ) and a rise again $\left(\Delta>40^{\circ}\right)$ in the northern hemisphere, although, the errors are relatively large here.

\subsection{Meridional Circulation from Inversions of Travel-times}

As presented in Section 3, we have implemented a raytheoretic scheme of inverting the travel time differences $\delta \tau_{\mathrm{NS}}$ to determine the stream function $\psi$, which allows the determination of both the flow components, $u_{\theta}$ and $u_{r}$, while satisfying the mass-conservation constraint. To illustrate the effect of choice of smoothing parameters we show the results obtained using two different values. The errors in the inverted results depend on the smoothing, with generally a higher smoothing leading to smaller errors. Results of thus determined $u_{\theta}$ and $u_{r}$ covering the whole of the convection zone in depth and within $\pm 60^{\circ}$ latitudes are shown in Figures 3 and 4 . The decreasing latitude extent for deeper layers are due to decreasing usable surface coverage of data for larger $\Delta$, i.e. we restrict measurements within $\pm 60^{\circ}$ latitudes to avoid errors due 


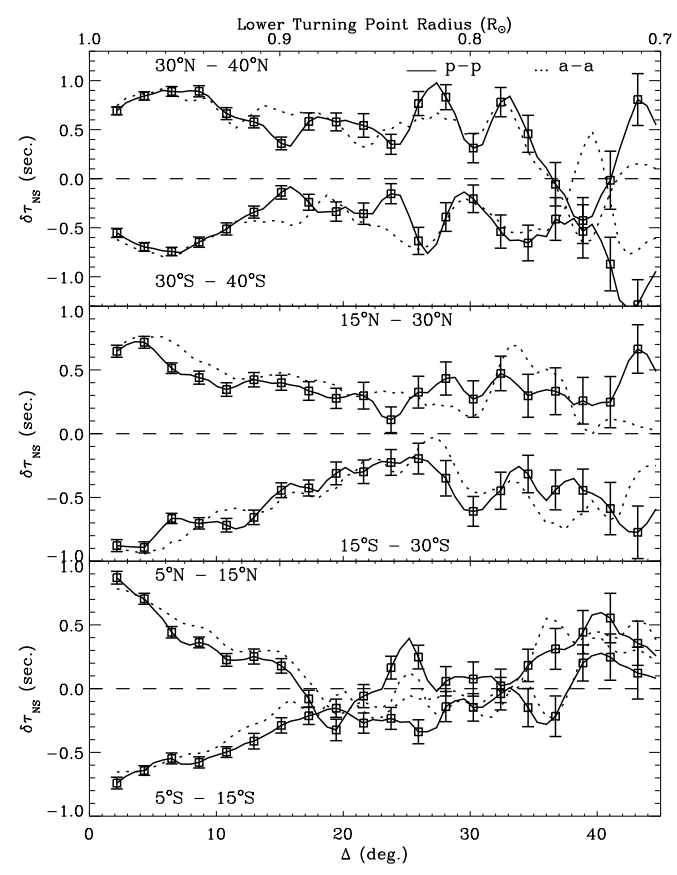

Figure 2. Travel-time differences, $\delta \tau_{\mathrm{NS}}$, averaged over three different latitude ranges, as marked in the panels, against the travel distance $\Delta$ and lower turning point radius.

to projection effects and this means that usable coverage reduces as $\Delta$ increases.

Variations over depth of inverted $u_{\theta}$ and $u_{r}$, averaged over the same ranges of latitudes used in Figure 2 for $\delta \tau_{\mathrm{NS}}$, are shown in right panels of Figures 3 and 4 . The lower smoothing lead to a slight increase in the variations in velocity profiles. These results show that the global large-scale MC has a possible return flow at depths below about $0.77 R_{\odot}$. This large single-cell $\mathrm{MC}$ is clear at higher latitudes. However, at lower latitudes (less than about $30^{\circ}$ ) there appear to be some sign change in $u_{\theta}$ around $0.9 R_{\odot}$, but the values are within error bars and these are also consistent with one deep cell of MC. Such variations look a little more pronounced in the case of solution with lower smoothing, as a comparison of Figures 3 and 4 would show. It can be seen that the results with two different smoothing shown in Figures 3 and 4 are not very different, thus showing that the solution is not particularly sensitive to choice of smoothing parameters in this range. Here we have only changed $\lambda_{\theta}$, but change in $\lambda_{r}$ also has similar effect. Nevertheless, below about $0.9 R_{\odot}$ the magnitude of velocity is comparable to errorbars and hence we cannot rule out the possibility of multiple cells, especially at lower latitudes. The depth variation of radial component $u_{r}$ too (see right panels of Figures 3 and 4), near the equatorial region, seem to indicate a tendency of sign change at about $0.9 R_{\odot}$, but again such changes are not significant considering the errors there. Zhao et al. (2013) found return flow below about $0.9 R_{\odot}$ and a second cell lower down. Considering the errorbars in inversion results, their results are roughly consistent with ours, though interpretation in terms of multiple cells is different. Although, the most recent study by Jackiewicz et al. (2015) using two years of data from GONG largely reproduces the shallow return flow below about $0.9 R_{\odot}$, it does not show the sec- ond cell of MC deeper down and instead shows strikingly anti-correlated flows in deeper layers between GONG and HMI results. It is important to note that the amplitudes of $u_{\theta}$, in general, gradually decrease below about $0.97 R_{\odot}$ in all results. Consistent inclusion of mass-conservation in our inversion process itself, is different from the results of both Zhao et al. (2013) and Jackiewicz et al. (2015). We believe this constitutes improved physical realism in our results than those of the above authors. We defer further discussion on the results to Section 5 .

Further, the higher-latitude poleward flows seen down to depths of about $70 \mathrm{Mm}$ (10\% of the solar radius from the surface) seem to drive deep downward flows beyond $40^{\circ}$ latitudes (refer to the right panel showing $u_{r}$ in Figure 3). This downward part of MC leads to the consistent signals of equator-ward return flow seen below about $0.77 R_{\odot}$ over the whole range of latitudes (left panel of Figure 4). Upwards flows at low latitudes and downward flows at higher latitudes, as captured in the inverted $u_{r}$ (right panels of Figure 3 and 4), peak at a depth of about $0.79 R_{\odot}$ with amplitudes of about $1 \mathrm{~m} \mathrm{~s}^{-1}$.

\subsection{Tests with Artificial Data}

In order to check if with the current level of errors in the travel-times it is possible to distinguish between the solution with single cell and that with a double cell, we generated artificial data with some assumed meridional flow profiles and added random errors to these which are consistent with those in observed data. Using these artificial data we did the inversions using the same (higher values) smoothing parameters to check for reliability of inversion results. Here we present results from two different meridional flow profiles, one a single cell extending till $0.7 R_{\odot}$ and another double cell profile with first cell ending at $0.80 R_{\odot}$. In both cases the surface velocity is chosen to be close to solar value to make meaningful comparison. The results are shown in Figures 5 .

The results for single cell profile are shown in the top row of Fig. 5] which compares the flow velocity in the input profile with those obtained by inversion of travel times. It can be seen that the agreement is fairly good and in most region the difference between the true value and inverted value of $u_{\theta}$ is less than $1 \mathrm{~m} \mathrm{~s}^{-1}$. The sign change in $u_{\theta}$ is also seen in the inverted profiles, though at a slightly deeper layer. Similarly, the bottom row of Fig. 5 shows the results for double cell profile of MC. In this case the agreement is even better than that for single cell profile. The inverted profiles clearly show two sign changes in $u_{\theta}$ with radius. Thus it is clear that our inversions are able to distinguish between the single and double cell profiles even in presence of observational errors. It may be noted that in both these cases the velocity in lower layers $\left(r<0.9 R_{\odot}\right)$ is rather small and still inversions can detect the signal reliably. The radial component of velocity, $u_{r}$ is very small for double cell pattern and it is difficult to determine it reliably in the deep interior, where it is of order of $0.1 \mathrm{~m} \mathrm{~s}^{-1}$.

These test results give some confidence in our results for the Solar MC and it is likely that solar MC is a single cell circulation pattern similar to what is assumed in flux transport dynamo models.

\section{DISCUSSION AND CONCLUSION}



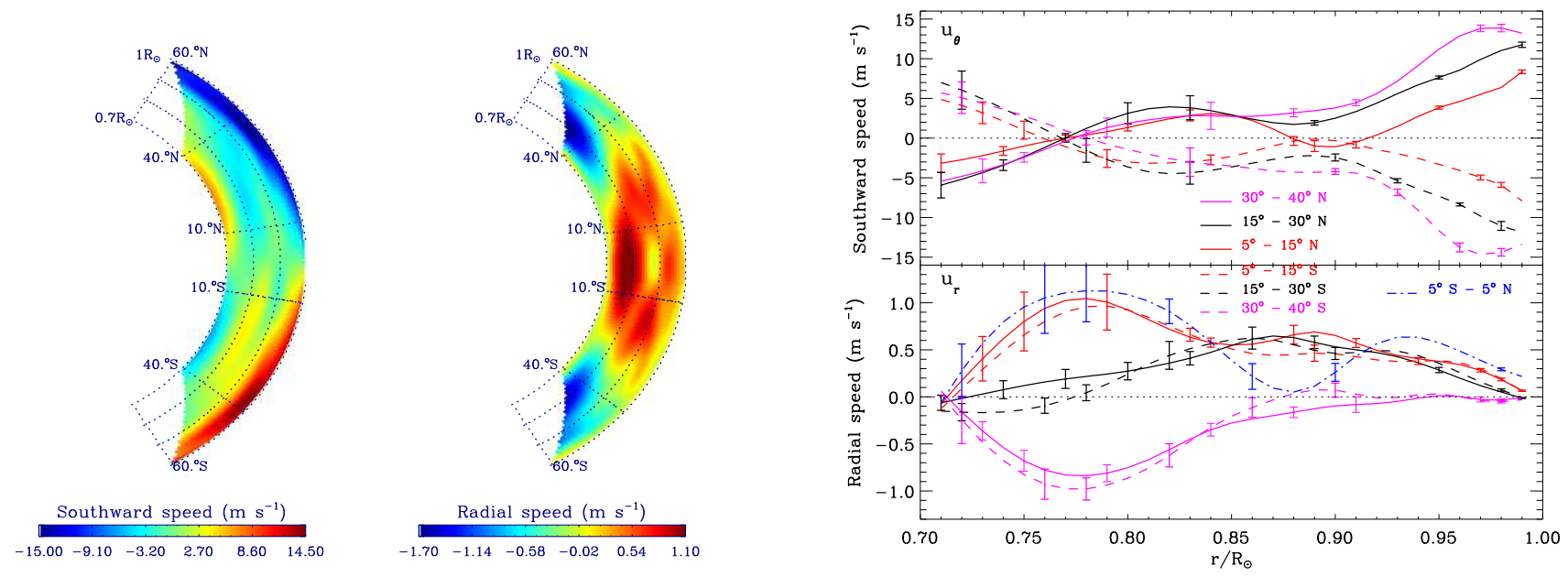

Figure 3. Meridional circulation that results from inversions of travel-time differences, $\delta \tau_{\mathrm{NS}}$ for the case of higher smoothing $\left(\lambda_{r}=\right.$ $\left.2 \times 10^{-4}, \lambda_{\theta}=5 \times 10^{-3}\right)$. The left part of the figure shows the $2 \mathrm{D}(r, \theta)$ profiles of $u_{\theta}$ and $u_{r}$. The two-panel plot on the right shows depth-profiles of $u_{\theta}$ and $u_{r}$ averaged over three different latitude ranges, as marked in the panel.
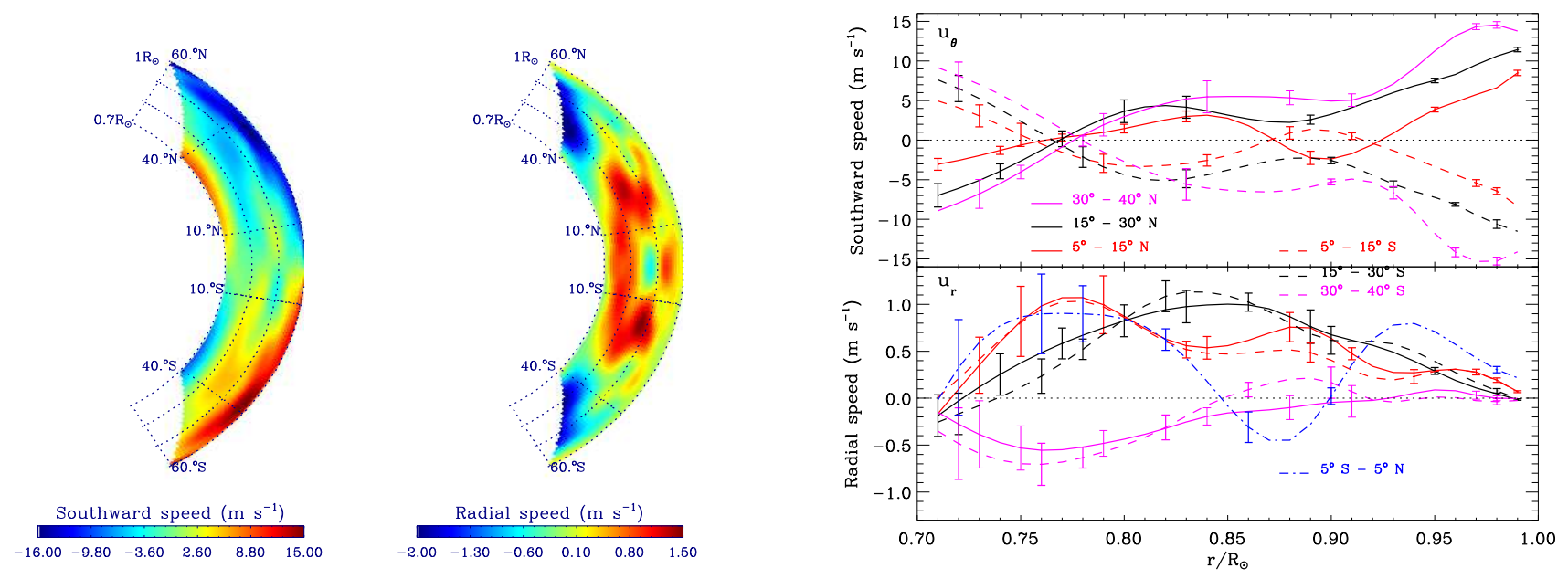

Figure 4. Same as Figure 3 but with a lower smoothing $\left(\lambda_{r}=2 \times 10^{-4}, \lambda_{\theta}=2 \times 10^{-3}\right)$.

Currently, there is an increased focus on helioseismic studies of the MC in the deep layers of the convection zone owing to several recent developments. Firstly, recent years have seen intense developments in modeling the solar dynamo owing to several curious features of the last few solar cycles, including the extended minimum between cycles 23 and 24 and the overall gradual decline of the strengths of the past few cycles (see Charbonneau (2014) and references therein). Secondly, almost two solar cycles long continuous helioseismic data is now available from GONG, MDI/SOHO and the new HMI/SDO, which can help in addressing solar interior variations related to the solar dynamo and the cycle. Temporal variations of large-scale motions in the interior of the Sun, e.g., the solar rotation and MC, can now be studied using these data. As far as the MC is concerned, measuring it over depth below about $10 \%$ of the solar radius from the surface is a difficult proposition as the expected signals in helioseismic observables are very small and are plagued by noise and systematics. As described in Section 1, local helioseismic methods, ring diagram and time-distance helioseismology, have successfully mapped the near-surface part of the MC. The identification and empirical correc- tion (or removal) for the large systematics, CLS, in helioseismic travel times by Zhao et al. (2012, 2013) have now made studying the MC throughout the convection zone possible albeit with contentious inferences on the deep structure of MC (Zhao et al. 2013; Jackiewicz et al. 2015).

Our work presented here provides an independent attempt to study $\mathrm{MC}$ in deep interior. We have made some improvements via adding additional physical constraints in the analysis procedures. As pointed out by Jackiewicz et al. (2015), the existing results on the deep MC (Zhao et al. 2013; Jackiewicz et al. 2015) are problematic in satisfying the mass-conservation constraints. The depth variation of inverted flow velocity amplitudes (refer to Figure 4 of Jackiewicz et al. (2015)) run counter to what is expected from mass-conservation. We believe that our analysis which addresses mass-conservation in deriving both the horizontal and radial flow components, $u_{\theta}$ and $u_{r}$, simultaneously from the inversion of measured travel times has added a significant improvement in the time-distance helioseismic probing of the deep MC. Within the limits of errors in the data and in the inversion procedure, we find that the MC has possibly a 

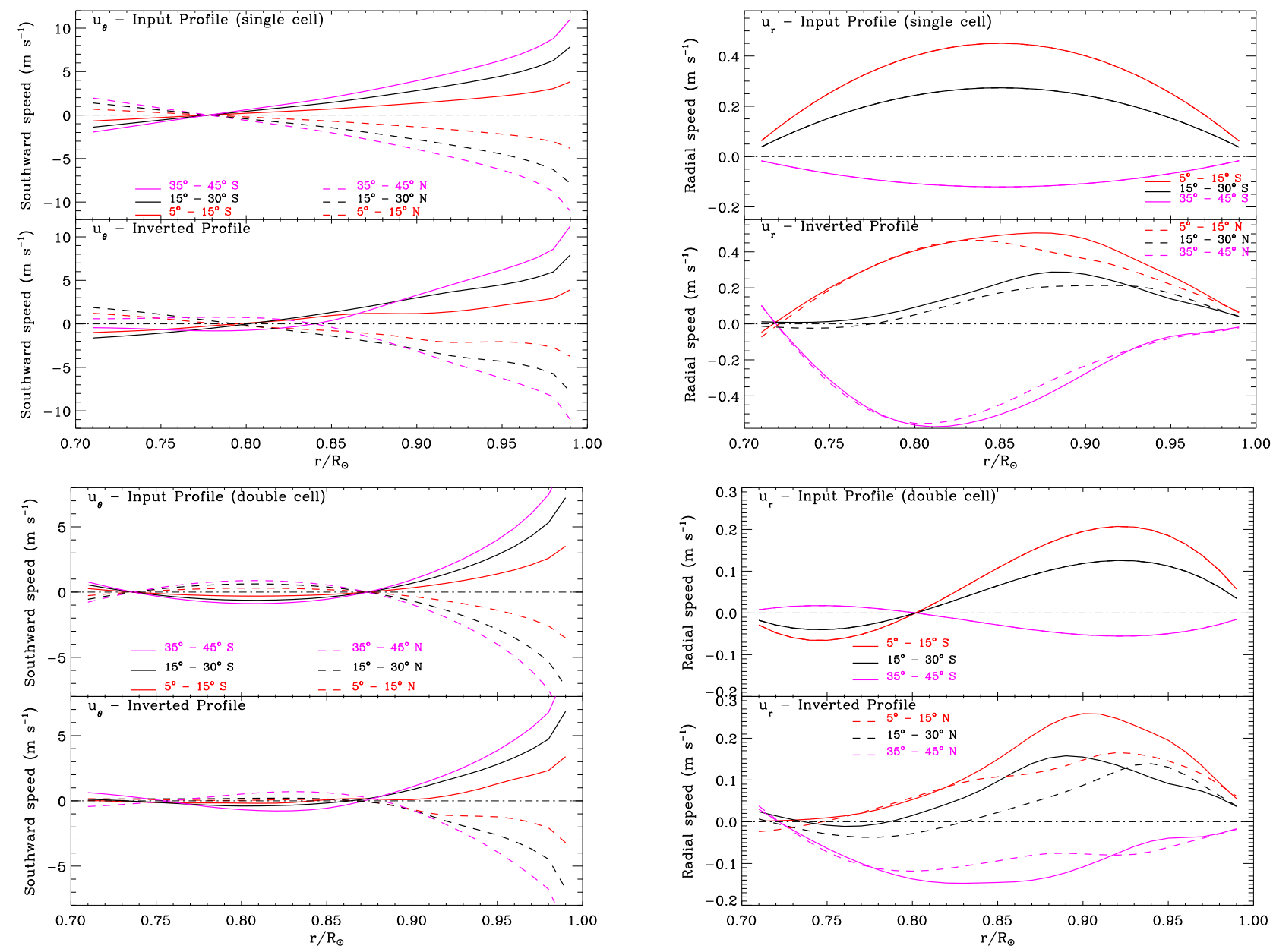

Figure 5. Cuts across depth of artificial data test inversions for $u_{\theta}$ and $u_{r}$ averaged over three different latitude ranges, as marked in the panel The upper part of the figure shows the meridional velocity components for artificial data for MC with single cell, while the lower part shows that for MC with double cell. The upper panels in each part show the input MC profile while the lower panels show the results obtained by inversion of artificial data. The errorbars are not shown in lower panel, but the errors are same as those shown in Figure 3 for observed data.

large-scale return flow at depths below about $0.77 R_{\odot}$. We do not see a shallow return flow at about $0.9 R_{\odot}$ consistently throughout the whole latitude range covered $\left( \pm 60^{\circ}\right)$, although, there are indications of sign reversals at latitudes less than about $30^{\circ}$, but not above our error limits, in both $u_{\theta}$ and $u_{r}$ at this depth. We find that the inverted $u_{r}$ exhibits broad upwellings extending over a depth range of $0.75 R_{\odot}-0.85 R_{\odot}$ within about $\pm 15^{\circ}$ latitudes with a peak amplitude of about $1 \mathrm{~m} \mathrm{~s}^{-1}$. At latitudes in the range of $30^{\circ}-40^{\circ}$ and beyond we find that the poleward flows with typical amplitudes of about $3-5 \mathrm{~m} \mathrm{~s}^{-1}$ over the depth range of $0.9 R_{\odot}-0.8 R_{\odot}$. This region is bounded by the deep downward flows beyond $40^{\circ}$ latitudes and forming a deep return flow at depths below $0.77 R_{\odot}$. Among the theoretical models or predictions in the literature, the $\mathrm{MC}$ profile calculated by Kitchatinov \& Olemskov (2011) from a mean-field hydrodynamical model appear to be close to our findings here. In particular, the sharp decline in $u_{\theta}$ observed over the depth range $0.97-0.9 R_{\odot}$ matches their model as well as the transition to equatorward flow seen beneath the depth of $0.77 R_{\odot}$. Numerical simulations of Featherstone \& Miesch (2015) show multiple cells of MC at low latitudes. Our inversion results also show some change in sign at low latitude, though the value is comparable to errorbars and is in a restricted range of latitude and depth.

To test if we could indeed detect multiple cells in $\mathrm{MC}$ we tried an exercise with artificial travel-time data generated using modeled single- and double-cell MC profiles. We find that it is indeed possible to detect multiple cells in $u_{\theta}$, even though the velocity in deep layers is comparable to errorbars. These results tend to suggest that the solar MC probably consists of a single-cell pattern covering the entire convection zone, although, significant improvements in analysis are required to confirm this result.

We thank Junwei Zhao (Stanford University) for helping us with the data preparation at the Stanford JSOC Helioseismology pipeline. The HMI data used are courtesy of NASA/SDO and the HMI science team. Data intensive computations performed in this work were carried out using the High-Performance Compute Cluster of the Indian Institute of Astrophysics, Bangalore. 


\section{REFERENCES}

Antia, H. M. 2012, Numerical Methods for Scientists and Engineers, Hindustan Book Agency, New Delhi

Baldner, C. S., \& Schou, J. 2012, ApJL, 760, L1

Basu, S., \& Antia, H. M. 2010, ApJ, 717, 488

Basu, S., Antia, H. M., \& Tripathy, S. 1999, ApJ, 512, 458

Charbonneau, P. 2010, Living Reviews in Solar Physics, vol.7, no.3

Charbonneau, P. 2014, Ann. Rev. Astron. Astrophys., 52, 251

Choudhuri, A. R., Schüssler, M., \& Dikpati, M. 1995, å, 303, L29

Dikpati, M. 2014, MNRAS, 438, 2380

Dikpati, M., \& Charbonneau, P. A. 1999, ApJ, 518, 508

Dikpati, M. \& Choudhuri, A. R. 1995, Sol. Phys., 161, 9

Dikpati, M., Gilman, P. A., de Toma, G., \& Ulrich, R. K. 2010, GeoRL, 37, L14107

Duvall, T. L. Jr 1979, Sol. Phys., 63, 3

Duvall, T. L., Jr., Jefferies, S. M., Harvey, J. W. \& Pomerantz, M. A. 1993, Nature, 362, 430

Duvall, T. L., Jr. 1995, in ASP Conf. Ser. 76, GONG 1994. Helioand Astro-Seismology from the Earth and Space, ed. R. K. Ulrich, E. J. Rhodes, Jr., \& W. Dappen (San Francisco, CA: ASP), 465

Eddington, A.S. 1925, The Observatory, 48, 73

Featherstone, Nicholas A., \& Miesch, Mark S. 2015, ApJ, 804, 67

Giles, P. M., Duvall, T. L., Jr., Scherrer, P. H., \& Bogart, R. S. 1997, Nature, 390, 52

Giles, P. M. 1999, PhD thesis, Stanford University.

Gilman, P. A. \& Miller, J. 1986, ApJS, 61, 585

González Hernández, I., Kholikov, S., Hill, F., Howe, R., \& Komm, R. 2008, Sol. Phys., 252, 235

Gough, D. O., Thompson, M. J. 1991, in Solar Interior and Atmosphere, University of Arizona Press, p. 519

Haber, D. A., Hindman, B. W., Toomre, J., et al. 2000, Sol. Phys., 192, 335

Haber, D. A., Hindman, B. W., Toomre, J., et al. 2002, ApJ, 570 855
Hanasoge, S.M., Duvall, T. L., Jr., \& DeRosa, M.L. 2010, ApJ, 712 , L98

Hathaway, D. H. 1996, ApJ, 460, 1027

Hathaway, D. H., \& Rightmire, L. 2010, Science, 327, 1350

Hathaway, D. H. 2012, ApJ, 760, 84

Jackiewicz, J., Serebryanskiy, A., \& Kholikov, S. 2015, ApJ, 805, 133

Kitchatinov, L. L., \& Olemskoy, S. V. 2011, MNRAS, 411, 1059

Kitchatinov, L. L., \& Rüdiger, G. 1999, å, 344, 911

Kosovichev, A.G. 1996, ApJL, 461, L55

Kosovichev, A.G., Duvall, T.L., Jr. in SCORe-'96: Solar Convection and Oscillations and Their Relationship, ed. F.P. Pijpers et al., 1997 (Kluwer) p. 241

Komm, R.W., Howard, R. F. \& Harvey, J.W. 1993, Sol. Phys., 147, 207

Lavely, E. M., Ritzwoller, M. H. 1992, Phil. Trans. Roy. Soc. Lon. A, 339,431

Miesch, M. S., \& Toomre, J. 2009, AnRFM, 41, 317

Rajaguru, S.P., Hughes, S.J., \& Thompson, M.J. 2004, Sol. Phys., 220,381

Rajaguru, S.P. 2008, arXiv:0802.2756

Schad, A, Timmer, J., Roth, M. 2013, ApJ, 778, L38

Scherrer, P. H., Schou, J., Bush, R. I., et al. 2012, Sol. Phys., 275, 207

Schou, J., \& Bogart, R.S. 1998, ApJL, 504, L131

Schou, J., Scherrer, P. H., Bush, R. I., et al. 2012, Sol. Phys., 275, 229

Ulrich, R. K. 2010, ApJ, 725, 658

von Zeipel, H. 1924, MNRAS, 84, 665

Wang, Y.-M., Nash, A.G. \& Sheeley, N.R., Jr. 1989, Science, 245, 712

Zhao, J., \& Kosovichev, A. G. 2004, ApJ, 603, 776

Zhao, J., Bogart, R.S., Kosovichev, A.G., Duvall, T.L.,Jr., \& Hartlep, T. 2013, ApJL, 774, L29

Zhao, J., Nagashima, K., Bogart, R. S., Kosovichev, A. G., \& Duvall, T. L., Jr. 2012, ApJL, 749, L5 